\title{
Comorbidity in polymyalgia rheumatica
}

\author{
C. Chatzigeorgiou, S.L. Mackie \\ Leeds Institute of Rheumatic and Musculoskeletal Medicine, University of Leeds, UK
}

\begin{abstract}
SUMMARY
Polymyalgia rheumatica (PMR) is the commonest inflammatory rheumatic disease affecting older people. The current mainstay of treatment is long-term oral glucocorticoid therapy. Management of these patients in clinical practice is often complicated by the presence of comorbidity. Comorbidity might be due to shared risk factors such as age, sex, or genetic background; to the presence of the disease itself; or to adverse effects of glucocorticoid therapy. Cardiovascular disease, osteoporosis/fracture, metabolic and ocular comorbidity are of particular interest to clinicians because of their relationship to glucocorticoid therapy and the relevance to clinical treatment decisions regarding glucocorticoid tapering. Patients at high risk of exacerbation of comorbidity by glucocorticoid therapy may be considered for adjunctive steroid-sparing therapies and thus may need specialist management. From a public health perspective, with the ageing population the prevalence of PMR is predicted to increase; accurate data on comorbidity will be needed for planning and delivery of healthcare services.
\end{abstract}

Key words: Polymyalgia rheumatica; Paraneoplastic syndrome; Cancer; Systematic review.

Reumatismo, 2018; 70 (1): 35-43

\section{INTRODUCTION}

olymyalgia rheumatica (PMR) presents with pain and stiffness of the shoulder and hip girdles in association with elevated laboratory markers of inflammation (1). The incidence of PMR is highest in the eighth decade of life; PMR is classically said not to occur under the age of 50 years. PMR may occur together with giant cell arteritis (GCA), a vasculitis of large and medium sized blood vessels $(2,3)$. There is no single diagnostic test for confirmation of PMR; other rheumatic conditions such as rheumatoid arthritis may sometimes present with similar features but the correct diagnosis usually emerges during follow-up (4-6). A systemic inflammatory response and arthralgia may also be the presenting feature of many other medical conditions, including cancer $(7,8)$. A challenge of interpretation of the current literature is the relative paucity of research literature compared to many other rheumatic diseases. Additionally, many early reports in the literature did not clearly distinguish well between PMR and GCA.

Patients with PMR and no evidence of oth- er rheumatic disease are generally treated with long-term oral glucocorticoids (GC) such as prednis(ol)one. The starting dose is $15-25 \mathrm{mg}$ of prednisone equivalent; this dose is gradually tapered over 1-2 years with an aim to stopping (9). Some patients however require ongoing GC therapy for many more years. There is some limited evidence for methotrexate as a steroidsparing drug (10). Older patients are particularly vulnerable to GC-related adverse effects, particularly where their baseline risk is already high due to pre-existing comorbidities.

Comorbidity in patients with PMR remains a significant issue in clinical management of individual patients with PMR, as well as being relevant to understanding the overall burden of this disease and its treatment within the general population. Recent clinical practice guidelines recommend assessment for comorbidity in all patients presenting with PMR (11). Comorbidity is important to establish in inflammatory rheumatic diseases because of increased prevalence of comorbid conditions compared to the general population (especially as a consequence of GC therapy), issues
Corresponding author:

Sarah Mackie

Leeds Institute of Rheumatic and

Musculoskeletal Medicine,

Level 8, Wellcome Trust Brenner Building,

St. James's University Hospital,

Leeds, LS9 7TF, UK

E-mail: s.l.mackie@leeds.ac.uk 
of equity (patients with chronic rheumatic diseases should have the same opportunities for health promotion/screening as people from the general population who do not have rheumatic disease) and the potential impact of comorbidities on outcomes of treatment of the rheumatic disease itself (12). In this review, we survey comorbid conditions that may be associated with PMR and those conditions which are particularly important for making decisions about continuing treatment.

\section{Co-occurence of polymyalgia rheumatica with giant cell arteritis}

GCA is associated with PMR in that the two conditions co-exist more frequently than would be expected by chance. The demographics of the two diseases are similar: older age, female sex, and northern European ancestry are risk factors for both PMR and GCA. Both PMR and GCA are characterised by a systemic inflammatory response which responds to systemic GC therapy (3, 13). The two conditions may either present at the same time or may sometimes be separated by long intervals; when presenting on different occasions, either one may present before the other (14). It has been postulated that both conditions may share a common genetic background. GCA has a strong association with genes within the major histocompatibility complex region (MHC) but as yet there are no large-scale studies of MHC associations in PMR (15-17).

Case reports and case series using modern imaging modalities such as positron emission tomography with low-dose CT (PET/ $\mathrm{CT}$ ) suggest that some patients apparently presenting with clinical features of isolated PMR additionally have inflammation of the aorta and proximal branches. This largevessel GCA may not be clinically evident without vascular imaging. Most case series report that polymyalgic symptoms are present in around half of patients diagnosed with GCA; in many cases the polymyalgic symptoms are the first symptom and the patient may be initially treated as isolated PMR before the more characteristic features of GCA appear (2).

The prevalence of subclinical GCA in pa- tients presenting with the clinical picture of PMR cannot be accurately determined without prospective vascular imaging studies; the prevalence of large-vessel GCA in patients with PMR is likely to be higher in patients recruited from specialist care than those diagnosed with PMR in the community. Estimates of the prevalence of GCA in patients with PMR can be as high as $21 \%$; patients with PMR and concomitant GCA may be older and have higher acute-phase markers in comparison with those with PMR and no $\operatorname{GCA}(6,14,18)$.

\section{Polymyalgic symptoms as a presenting feature of other rheumatic diseases}

Due to the non-specific nature of polymyalgic symptoms, other inflammatory rheumatic diseases may often be initially diagnosed as PMR (19). Rheumatoid arthritis (which in this age group is sometimes called late-onset rheumatoid arthritis or LORA) may frequently present with features identical to PMR; the characteristic swelling of the small joints may only manifest as the GC dose is tapered over the first year (20). With the advent of modern diagnostic imaging in routine clinical practice it has been proposed that imaging may help identify other inflammatory rheumatic diseases at an early stage, before starting GC treatment (21). Further studies are required to formally test this idea.

\section{Polymyalgic symptoms as a presenting feature of malignancy}

Malignancy can present with PMR-like symptoms (22); epidemiological studies are contradictory $(7,23,24)$ but there is some evidence for an increase in cancer diagnoses following a diagnosis of PMR, particularly during the first six months following the PMR diagnosis (25). Prior to this, there were a few case reports of PMR occurring in patients with malignancy; it has been suggested that clinicians might have a higher index of suspicion for malignancy in patients with atypical features of PMR such as absence of prolonged morning stiffness, non-response to GC and/or absence of typical PMR imaging 
findings (26). In a systematic review and meta-analysis, Ungprasert et al. reported a slightly elevated malignancy risk among patients with GCA/PMR, with a higher risk for malignancy during the first year after the diagnosis of PMR. However, it should be noted that in some of the studies included in this meta-analysis, it was difficult to separate out patients with isolated PMR from those with a diagnosis of GCA in addition to PMR. Furthermore, the authors' funnel plot suggested the possibility of publication bias. One of the studies included in the meta-analysis only included hospitalized patients, potentially introducing a selection bias; if the study restricted to hospitalized cases was excluded from the analysis, statistical significance of the pooled risk ratio was lost (27). It has been recently proposed that older age, male sex and more extensive joint involvement should be considered as potential red flags for cancer in PMR (28). In nationwide registry studies, patients with PMR appeared to be at elevated risk for lymphoma, both for Hodgkin's and non-Hodgkin's lymphoma overall $(8,29)$ as well as for some subtypes of haematological malignancy $(30,31)$.

\section{Comorbidity associated with prior glucocorticoid exposure}

Comorbidity in PMR may be particularly important where patients have already had extensive prior exposure to GC, for example patients with long-standing chronic respiratory disease (such as asthma, bronchiectasis or chronic obstructive pulmonary disease) requiring multiple previous courses of oral GC over the years prior to their diagnosis of PMR. Many GC-related adverse events are related to cumulative dose of GC; osteoporosis, for example, is common in patients who have been treated with GC for chronic respiratory disease. If the baseline fracture risk before PMR is already high, then adding in long-term GC for the PMR may lead to a high absolute fracture risk. Such patients may need careful management to minimize the cumulative dose of GC required for their PMR.

\section{Glucocorticoid-related adverse events in} polymyalgia rheumatica

Case series and disease cohorts of patients with PMR reveal a high incidence of glucocorticoid-related adverse events including osteoporosis, fracture, diabetes, weight gain, hypertension, diabetes, myocardial infarction and stroke (32-37). This in itself is important for making treatment decisions regarding adjustment of GC therapy, or commencement steroid sparing therapy, in PMR. However, without some comparison to controls without PMR, these study designs do not necessarily indicate an elevated risk of these adverse events in individuals with PMR over and above individuals without PMR of the same age and sex. Well-powered studies involving comparison to population-based controls have been conducted in regard to certain of these comorbidities, notably cardiovascular disease and osteoporosis.

\section{Cardiovascular disease as a comorbidity in polymyalgia rheumatica}

It has been hypothesised that, like other inflammatory rheumatic diseases such as RA and ankylosing spondylitis, PMR may be associated with cardiovascular disease due to accelerated atherosclerosis. There are various plausible biological mechanisms by which systemic inflammation may cause endothelial dysfunction and/or drive accelerated atherosclerosis. Although endothelial dysfunction can be studied experimentally (38) and does appear to be associated with untreated PMR, epidemiological studies are required to test whether treated PMR is associated with an excess of cardiovascular disease/cardiovascular events, and whether any association persists after controlling for traditional cardiovascular risk factors. Here, GC treatment is a complicating factor; although GC therapy may control the systemic inflammation, GC excess (Cushing's syndrome) is a known cause of accelerated cardiovascular disease $(39,40)$. Cushing's syndrome is associated with an increased risk of hypertension, diabetes and dyslipidaemia.

The absolute risk of cardiovascular disease attributable to GC therapy in rheumatic dis- 
ease remains somewhat controversial (41). According to a recent systematic review an association between PMR and vascular disease was reported by some studies, but this was more commonly reported in retrospective studies compared to prospective studies (42). A meta-analysis suggested a significantly elevated risk ratio for coronary artery disease in patients with PMR compared to patients without PMR; however, high statistical heterogeneity between studies was identified so this pooled risk ratio must be interpreted with caution (43). There may be an increased risk of subsequent stroke in patients with PMR compared to patients without PMR $(44,45)$. Two different studies of patients without cardiovascular disease from the UK reported divergent findings regarding risk of cardiovascular disease following PMR diagnosis $(46,47)$; this difference in findings may relate to differences in the datasources used as well as potential differences in coding and different statistical analyses. At present, therefore, due to limitations of currently available data it remains unclear whether treated PMR is associated with an elevated long-term cardiovascular risk.

\section{Osteoporosis as a comorbidity in polymyalgia rheumatica}

Fragility fracture is a well-known risk of long-term GC therapy. This is particularly evident for prednis(ol)one doses $>5 \mathrm{mg}$ per day (48). Fracture risk appears to relate both to the daily dose and to the overall duration of GC treatment. It has been suggested that the highest fracture risk in PMR is during the first six months of therapy, when the GC dose is highest (49); however, in other studies the fracture risk appears greatest after prolonged therapy, indicating the importance of treatment duration (33). In a meta-analysis, Hoes et al. reported that higher dosages of GCs resulted in higher adverse event rates in studies of PMR of comparable quality (50). In a recent multicentre study, Rossini et al. noted that fragility fractures, such as vertebral fractures, were common in patients treated for PMR despite anti-resorptive therapy in $80 \%$, although only $10 \%$ of patients with PMR de- veloped new fractures, with a further $37 \%$ already having a prior history of fragility fracture prior to treatment of PMR (51). In a retrospective study of 222 patients with PMR, Mazzantini et al. reported that osteoporosis and fragility fractures occurred significantly more frequently in those who were treated for $>2$ years compared to those treated for $<2$ years (33). After adjustment for age and sex, fragility fractures were significantly associated with cumulative GC dose, and osteoporosis was significantly associated with GC treatment duration (33).

For the management of glucocorticoidinduced osteoporosis, all patients need an adequate intake of calcium and vitamin D either through the diet or through supplements, and appropriate advice on physical activity and exercise. Mok et al., in a randomized, placebo-controlled trial of 120 patients receiving high-dose steroid therapy $(>0.5 \mathrm{mg} / \mathrm{kg} /$ day of oral prednisolone or its equivalent for at least 6 weeks) observed that the group of patients who were using bisphosphonates (risedronate $5 \mathrm{mg}$ /day) had significant gain in spinal bone mineral density $(+0.7 \pm 0.3 \%)$ whereas a fall in bone mineral density was observed in the placebo group $(-0.7 \pm 0.4 \%)$ (52).

Regarding further pharmacological treatments, many local and national guidelines in the UK, US and France provide recommendations appropriate for the local healthcare setting. The American College of Rheumatology has recently published an updated, evidence-based guidelines on prevention and management of glucocorticoid-induced osteoporosis (53). Recommendations in this paper, include treating with calcium and vitamin $\mathrm{D}$ in adults at low fracture risk, treating with calcium and vitamin D plus an additional osteoporosis medication (oral bisphosphonate preferred) in adults at moderate-to-high fracture risk, continuing calcium plus vitamin $\mathrm{D}$ but switching from an oral bisphosphonate to another antifracture medication in adults in whom oral bisphosphonate treatment is not appropriate, and continuing oral bisphosphonate treatment or switching to another antifracture medication in adults who com- 
plete a planned oral bisphosphonate regimen but continue to receive GC treatment. The commonly-utilised World Health Organisation FRAX tool now allows clinicians to account for glucocorticoid therapy in estimating fracture risk, but it needs to be acknowledged that the fracture risk is dose-dependent and that further adjustment to risk estimates may be appropriate to account for the level of glucocorticoid dose, since patients receiving greater than $7.5 \mathrm{mg}$ prednisolone daily are at a higher risk of fracture than those receiving less than 7.5 mg daily.

In addition GC therapy is a well-documented risk factor for avascular necrosis, which may occur in various sites including the femoral head (54). Because avascular necrosis is relatively rare compared to fragility fracture, and may not be well-recorded in population-based datasets, the precise risk of avascular necrosis in PMR is unknown. However, it is important because it may require surgical intervention.

\section{Metabolic comorbidity in polymyalgia rheumatica}

Long-term GC in the context of PMR is associated with weight gain in almost all patients. In 129 patients with PMR, 43.5\% gained $>5 \%$ body weight by 12 months (32). In addition systemic GC have a more immediate adverse effect on glucose tolerance and may induce hyperglycaemia and diabetes mellitus $(34,49)$. Gabriel et al. reported that the risk ratio of diabetes was 2.0 in males and 2.2 in females with PMR when compared with age- and sex-matched controls from the same population (49). A meta-analysis revealed that metabolic disease is one of the most frequently-reported adverse events in PMR (50). The precise risk of diabetes in GC-treated PMR is unclear.

As well as increasing the risk of diabetes, GC-induced weight gain can exacerbate many other comorbidities and result in a significant health burden including exertional dyspnoea, joint pains, acid reflux, hypertension, psychological distress and all the other potential consequences of obesity. GC-induced weight gain can lead to increased symptoms from osteoarthritis of the weight-bearing joints. This can be particularly problematic because the antiinflammatory action of GC may also alleviate some of the symptoms of osteoarthritis, leading to an increase in joint pains on GC tapering that may be mistaken for a flare of PMR and prompting escalation of GC therapy.

\section{Ocular comorbidity in polymyalgia rheumatica}

Cataract is common in older people. Longterm GC therapy can lead to acceleration of cataract formation (55), particularly posterior subcapsular cataract. Especially in patients with pre-existing visual impairment from other age-related conditions (such as macular degeneration), accelerated cataract can severely impair patient quality of life. Cataract may require surgical treatment to avoid progression to blindness. There is some evidence that patients with GC-treated PMR are at increased risk of cataract (56) although the research base does not enable us to make a precise estimate of the degree of elevation of cataract risk in PMR compared to the general population.

\section{Iatrogenic adrenal suppression}

Patients with untreated PMR may have impaired functioning of the hypothalamicpituitary-adrenal (HPA) axis (57-59). This has been suggested to be possibly due to their age combined with the effect of the systemic inflammation that accompanies untreated PMR. During long-term GC therapy, however, homeostatic mechanisms cause iatrogenic suppression of cortisol production by the adrenal glands. This adrenal suppression may persist even after the exogenous GC therapy has ceased. The result of this is that during tapering of the GC dose in PMR, it may take some time for the HPA axis to recover its former responsiveness. How this should be managed in clinical practice remains unclear. Patients with iatrogenic adrenal suppression receiving subphysiological doses of GC may require short-term rescue GC therapy in the event of severe intercurrent illness or major surgery. 


\section{Psychological comorbidity}

Psychological sequelae are common following the diagnosis of any chronic illness. Compounding this, GC therapy itself may have psychological adverse effects ranging from anxiety/irritability to significant depression or, rarely, frank psychosis. The reported prevalence of depression in PMR varies from 2 to $29 \%$ depending on the method of ascertainment of depression used (32, 60-62).

The sample size of those studies was relatively small and were based on secondary care settings, thus limiting generalisability. In a recent cross-sectional study in primary care settings in UK, Vivekanantham et al. reported a higher prevalence of current depressive symptoms among primary care PMR patients in comparison with the general older adult population (63). Risk factors for depression in this study included female sex, younger age, and presence of glucocorticoid-related comorbidities; in multivariable analysis, only diabetes remained significantly associated with symptoms of depression (63).

\section{DISCUSSION} AND CONCLUSIONS

Clinical practice guidelines recommend assessment for comorbidity in PMR, with a particular focus on those comorbidities that are relevant to decisions about GC dose and duration. In this review, we focus on cardiovascular disease, osteoporosis, metabolic disease/diabetes, cataract and depression, and the management of iatrogenic adrenal suppression in the context of tapering long-term GC therapy. Standardised reporting forms have been suggested for assessment of comorbidity in patients with inflammatory rheumatic diseases in daily clinical practice and would also appear relevant to the clinical care of patients with PMR (12).

PMR symptoms may also herald the onset of other serious diseases such as GCA, RA or malignancy. The relatively high rate of diagnosis of these other diseases during the first year of treatment for PMR suggests that there should be a low threshold for in- vestigation if the disease does not appear to be following its usual course. Patients with a dual diagnosis of PMR and GCA are generally treated with high-dose GC because higher doses of GC are required for GCA than for PMR. Patients with polymyalgic symptoms who subsequently develop clinical features of RA may often respond well to a combination of standard RA therapy together with low-dose GC therapy until the inflammation comes under effective control; this presentation of RA is however sufficiently uncommon that large-scale population data on this are lacking. How or whether to combine ongoing GC therapy for PMR with treatment of a newly-diagnosed cancer remains unclear, and decisions need to be taken on an individualized basis depending on the type of cancer and the patient's symptom burden.

Accurate estimates of risk and prognosis of comorbidities accompanying PMR are difficult to obtain from traditional trials and disease cohorts because of limitations of sample size and the observation that many important GC-related adverse events, such as fracture, tend to accumulate over many years of GC therapy. Increased availability of routinely-collected clinical data, particularly with linkage across multiple datasets, may help to derive more precise estimates as to the prognosis of patients with PMR and comorbid conditions and to help inform how we should screen for, monitor and manage these in routine clinical practice.

Conflict of interest: Sarah L. Mackie has served on two Medical Advisory Boards for Roche/Chugai and has received research funding from Vasculitis UK, and Fight for Sight. Previously she has received funding from the Wellcome Trust and Research into Ageing. She is a Trustee of the UK charity PMRGCAuk. The authors alone are responsible for the content and writing of this article.

Funding: Sarah L. Mackie is funded by a National Institute for Health Research (NIHR) Clinician Scientist Fellowship. The views expressed are those of the au- 
thors and not necessarily those of the National Health Service, NIHR or the Department of Health.

\section{REFERENCES}

1. Nesher G. Polymyalgia rheumatica-diagnosis and classification. J Autoimmun. 2014; 4849: 76-8.

2. Salvarani C, Cantini F, Hunder GG. Polymyalgia rheumatica and giant-cell arteritis. Lancet. 2008; 372: 234-45.

3. Buttgereit F, Dejaco C, Matteson EL, Dasgupta B. Polymyalgia rheumatica and giant cell arteritis: a systematic review. JAMA. 2016; 315: 2442-58.

4. Gonzalez-Gay MA, Garcia-Porrua C, Salvarani $\mathrm{C}$, et al. The spectrum of conditions mimicking polymyalgia rheumatica in Northwestern Spain. J Rheumatol. 2000; 27: 2179-84.

5. Gonzalez-Gay MA, Garcia-Porrua C, Salvarani $\mathrm{C}$, et al. Polymyalgia manifestations in different conditions mimicking polymyalgia rheumatica. Clin Exp Rheumatol. 2000; 18: 755-9.

6. Salvarani C, Gabriel SE, O'Fallon WM, Hunder GG. Epidemiology of polymyalgia rheumatica in Olmsted County, Minnesota, 19701991. Arthritis Rheum. 1995; 38: 369-73.

7. Pfeifer EC, Crowson CS, Major BT, Matteson EL. Polymyalgia rheumatica and its association with cancer. Rheumatology (Sunnyvale). 2015; (Suppl. 6).

8. Fallah M, Liu X, Ji J, et al. Hodgkin lymphoma after autoimmune diseases by age at diagnosis and histological subtype. Ann Oncol. 2014; 25: 1397-404.

9. Dasgupta B, Cimmino MA, Kremers HM, et al. 2012 Provisional classification criteria for polymyalgia rheumatica: a European League Against Rheumatism/American College of Rheumatology collaborative initiative. Arthritis Rheum. 2012; 64: 943-54.

10. Dejaco C, Singh Y, Perel P, et al. Current evidence for therapeutic interventions and prognostic factors in polymyalgia rheumatica: a systematic literature review informing the 2015 European League Against Rheumatism/ American College of Rheumatology recommendations for the management of polymyalgia rheumatica. Ann Rheum Dis. 2015; 74: 1808-17.

11. Dejaco C, Singh Y, Perel P, et al. 2015 Recommendations for the management of polymyalgia rheumatica: a European League Against Rheumatism/American College of Rheumatology Collaborative Initiative. Arthritis Rheumatol. 2015; 67: 2569-80.

12. Baillet A, Gossec L, Carmona L, et al. Points to consider for reporting, screening for and preventing selected comorbidities in chronic inflammatory rheumatic diseases in daily practice: a EULAR initiative. Ann Rheum Dis. 2016; 75: 965-73.

13. Pipitone N, Salvarani C. Update on polymyalgia rheumatica. Eur J Intern Med. 2013; 24: 583-9.

14. Crowson CS, Matteson EL. Contemporary prevalence estimates for giant cell arteritis and polymyalgia rheumatica, 2015. Semin Arthritis Rheum. 2017 [Epub ahead of print].

15. Cantini F. Are polymyalgia rheumatica and giant cell arteritis the same disease? Semin Arthritis Rheum. 2004; 33: 294-301.

16. Carmona FD, Gonzalez-Gay MA, Martin J. Genetic component of giant cell arteritis. Rheumatology. 2014; 53: 6-18.

17. Gonzalez-Gay MA, Amoli MM, Garcia-Porrua $\mathrm{C}$, Ollier WE. Genetic markers of disease susceptibility and severity in giant cell arteritis and polymyalgia rheumatica. Semin Arthritis Rheum. 2003; 33: 38-48.

18. Franzen P, Sutinen S, von Knorring J. Giant cell arteritis and polymyalgia rheumatica in a region of Finland: an epidemiologic, clinical and pathologic study, 1984-1988. J Rheumatol. 1992; 19: 273-6.

19. Pease CT, Haugeberg G, Morgan AW, et al. Diagnosing late onset rheumatoid arthritis, polymyalgia rheumatica, and temporal arteritis in patients presenting with polymyalgic symptoms. A prospective longterm evaluation. J Rheumatol. 2005; 32: 1043-6.

20. Caporali R, Montecucco C, Epis O, et al. Presenting features of polymyalgia rheumatica (PMR) and rheumatoid arthritis with PMRlike onset: a prospective study. Ann Rheum Dis. 2001; 60: 1021-4.

21. Falsetti P, Acciai C, Volpe A, Lenzi L. Ultrasonography in early assessment of elderly patients with polymyalgic symptoms: a role in predicting diagnostic outcome? Scand J Rheumatol. 2011; 40: 57-63.

22. Muller S, Hider S, Helliwell T, Partington R, Mallen $\mathrm{C}$. The real evidence for polymyalgia rheumatica as a paraneoplastic syndrome. Reumatismo 2017; 70: 23-34.

23. Myklebust G, Wilsgaard T, Jacobsen BK, Gran JT. No increased frequency of malignant neoplasms in polymyalgia rheumatica and temporal arteritis. A prospective longitudinal study of 398 cases and matched population controls. J Rheumatol. 2002; 29: 2143-7.

24. Haga HJ, Eide GE, Brun J, et al. Cancer in association with polymyalgia rheumatica and temporal arteritis. J Rheumatol. 1993; 20: 1335-9.

25. Muller S, Hider SL, Belcher J, et al. Is cancer associated with polymyalgia rheumatica? A cohort study in the General Practice Research Database. Ann Rheum Dis. 2014; 73: 1769-73. 
26. Niccoli L, Salvarani C, Baroncelli G, et al. Renal cell carcinoma mimicking polymyalgia rheumatica. Clues for a correct diagnosis. Scand J Rheumatol. 2002; 31: 103-6.

27. Ungprasert P, Sanguankeo A, Upala S, Knight EL. Risk of malignancy in patients with giant cell arteritis and polymyalgia rheumatica: a systematic review and meta-analysis. Semin Arthritis Rheum. 2014; 44: 366-70.

28. Bellan M. Association between rheumatic diseases and cancer: results from a clinical practice cohort study. Intern Emerg Med. 2017 [Epub ahead of print].

29. Fallah M, Liu X, Ji J, et al. Autoimmune diseases associated with non-Hodgkin lymphoma: a nationwide cohort study. Ann Oncol. 2014; 25: 2025-30.

30. Kristinsson SY. Immune-related and inflammatory conditions and risk of lymphoplasmacytic lymphoma or Waldenstrom macroglobulinemia. J Natl Cancer Inst. 2010; 102: 557-67.

31. Kristinsson SY, Landgren O, Samuelsson J, et al. Autoimmunity and the risk of myeloproliferative neoplasms. Haematologica. 2010; 95: 1216-20.

32. Hutchings A. Clinical outcomes, quality of life, and diagnostic uncertainty in the first year of polymyalgia rheumatica. Arthritis Rheum. 2007; 57: 803-9.

33. Mazzantini M. Adverse events during longterm low-dose glucocorticoid treatment of polymyalgia rheumatica: a retrospective study. J Rheumatol. 2012; 39: 552-7.

34. Bahlas S, Ramos-Remus C, Davis P. Clinical outcome of 149 patients with polymyalgia rheumatica and giant cell arteritis. J Rheumatol. 1998; 25: 99-104.

35. Faez S, Lobo AM, Unizony SH, et al. Ocular inflammatory disease in patients with polymyalgia rheumatica: A case series and review of the literature. Clin Rheumatol. 2016; 35: 251-8.

36. Corrao S, Pistone G, Scaglione R, et al. Fast recovery with etanercept in patients affected by polymyalgia rheumatica and decompensated diabetes: a case-series study. Clin Rheumatol. 2009; 28: 89-92.

37. Arai T, Tanaka R, Kaburaki T. Ocular inflammation associated with polymyalgia rheumatica without concomitant giant-cell arteritis: a report of three cases. Ocul Immunol Inflamm. 2017: 1-4.

38. Pirro M. Imbalance between endothelial injury and repair in patients with polymyalgia rheumatica: improvement with corticosteroid treatment. J Intern Med. 2012; 272: 177-84.

39. Girod JP, Brotman DJ. Does altered glucocorticoid homeostasis increase cardiovascular risk? Cardiovasc Res. 2004; 64: 217-26.

40. Schacke H, Docke WD, Asadullah K. Mechanisms involved in the side effects of glucocorticoids. Pharmacol Ther. 2002; 96: 23-43.
41. Davis JM, 3rd, Maradit-Kremers H, Gabriel SE. Use of low-dose glucocorticoids and the risk of cardiovascular morbidity and mortality in rheumatoid arthritis: what is the true direction of effect? J Rheumatol. 2005; 32: 1856-62.

42. Hancock AT, Mallen CD, Belcher J, Hider SL. Association between polymyalgia rheumatica and vascular disease: a systematic review. Arthritis Care Res (Hoboken). 2012; 64: 1301-5.

43. Ungprasert P, Koster MJ, Warrington KJ, Matteson EL. Polymyalgia rheumatica and risk of coronary artery disease: a systematic review and meta-analysis of observational studies. Rheumatol Int. 2017; 37: 143-9.

44. Kang JH, Sheu JJ, Lin HC. Polymyalgia rheumatica and the risk of stroke: a three-year follow-up study. Cerebrovasc Dis. 2011; 32: 497-503.

45. Zoller B, Li X, Sundquist J, Sundquist K. Risk of subsequent ischemic and hemorrhagic stroke in patients hospitalized for immune-mediated diseases: a nationwide follow-up study from Sweden. BMC Neurol. 2012; 12: 41.

46. Hancock AT. Risk of vascular events in patients with polymyalgia rheumatica. CMAJ. 2014; 186: E495-501.

47. Pujades-Rodriguez M, Duyx B, Thomas SL, et al. Associations between polymyalgia rheumatica and giant cell arteritis and 12 cardiovascular diseases. Heart. 2016; 102: 383-9.

48. Pearce G, Ryan PF, Delmas PD, et al. The deleterious effects of low-dose corticosteroids on bone density in patients with polymyalgia rheumatica. Br J Rheumatol. 1998; 37: 292-9.

49. Gabriel SE, Sunku J, Salvarani C, et al. Adverse outcomes of antiinflammatory therapy among patients with polymyalgia rheumatica. Arthritis Rheum. 1997; 40: 1873-8.

50. Hoes JN, Jacobs JW, Verstappen SM, et al. Adverse events of low- to medium-dose oral glucocorticoids in inflammatory diseases: a meta-analysis. Ann Rheum Dis. 2009; 68: 1833-8.

51. Rossini M. Prevalence and incidence of osteoporotic fractures in patients on long-term glucocorticoid treatment for rheumatic diseases: the Glucocorticoid Induced OsTeoporosis TOol (GIOTTO) study. Reumatismo. 2017; 69: 30-9.

52. Mok CC, Tong KH, To CH, et al. Risedronate for prevention of bone mineral density loss in patients receiving high-dose glucocorticoids: a randomized double-blind placebo-controlled trial. Osteoporos Int. 2008; 19: 357-64.

53. Buckley L. American College of Rheumatology guideline for the prevention and treatment of glucocorticoid-induced osteoporosis. Arthritis Care Res (Hoboken). 2017; 69: 1095-110.

54. Aaron RK, Voisinet A, Racine J, et al. Corti- 
costeroid-associated avascular necrosis: dose relationships and early diagnosis. Ann N Y Acad Sci. 2011; 1240: 38-46.

55. Curtis JR. Population-based assessment of adverse events associated with long-term glucocorticoid use. Arthritis Rheum. 2006; 55: 420-6.

56. Gabriel SE, Sunku J, Salvarani C, et al. Adverse outcomes of antiinflammatory therapy among patients with polymyalgia rheumatica. Arthritis Rheum. 1997; 40: 1873-8.

57. Demir H. Evaluation of the hypothalamic-pituitary-adrenal axis in untreated patients with polymyalgia rheumatica and healthy controls. Scand J Rheumatol. 2006; 35: 217-23.

58. Straub RH. The adrenal steroid status in relation to inflammatory cytokines (interleukin-6 and tumour necrosis factor) in polymyalgia rheumatica. Rheumatology. 2000; 39: 624-31.

59. Cutolo M, Straub RH. Polymyalgia rheumatica: evidence for a hypothalamic-pituitary-ad- renal axis-driven disease. Clin Exp Rheumatol. 2000 ; 18: 655-8.

60. Dasgupta B, Dolan AL, Panayi GS, Fernandes L. An initially double-blind controlled 96 week trial of depot methylprednisolone against oral prednisolone in the treatment of polymyalgia rheumatica. $\mathrm{Br} \mathrm{J}$ Rheumatol. 1998; 37: 189-95.

61. Kimura M. Clinical characteristics of patients with remitting seronegative symmetrical synovitis with pitting edema compared to patients with pure polymyalgia rheumatica. J Rheumatol. 2012; 39: 148-53.

62. Chuang TY, Hunder GG, Ilstrup DM, Kurland LT. Polymyalgia rheumatica: a 10-year epidemiologic and clinical study. Ann Intern Med. 1982; 97: 672-80.

63. Vivekanantham A, Blagojevic-Bucknall M, Clarkson $\mathrm{K}$, et al. How common is depression in patients with polymyalgia rheumatica? Clin Rheumatol. 2017 [Epub ahead of print]. 